Thaiane Moleta Vargas ${ }^{1}$

Doutoranda em Ciências

Sociais Aplicadas pela UEPG.

E-mail:

thaiane_moleta@yahoo.com.br

\section{Leandro Martinez}

Vargas $^{1}$

Doutor em Educação Física UNICAMP, Professor do

Departamento de Educação

Física da UEPG. Email:

leandro.vargas@uol.com.br

\section{José Roberto Herrera}

\section{Cantorani ${ }^{3}$}

Doutor em Educação Física -

UNICAMP, Professor do Instituto

Federal de Educação, Ciência e

Tecnologia de São Paulo (IFSP)

e docente permanente do

Programa de Pós-Graduação

em Ensino de Ciência e

Tecnologia (PPGECT) da

UTFPR. E-mail:

cantorani@yahoo.com.br

\section{Bruno Pedroso ${ }^{1}$}

Doutor em Educação Física -

UNICAMP. Docente permanente

nos Programas de Pós-

Graduação em Ciências Sociais

Aplicadas e Ciências da Saúde da UEPG. E-mail:

prof.brunopedroso@gmail.com

\section{QUALIDADE DE VIDA EM INGRESSANTES E CONCLUINTES DE DIFERENTES CURSOS UNIVERSITÁRIOS}

\author{
QUALITY OF LIFE FOR FRESHMEN AND GRADUATES OF \\ DIFFERENT UNIVERSITY COURSES
}

\section{RESUMO}

O presente trabalho teve por objetivo avaliar a percepção da QV de estudantes universitários, comparando as informações obtidas de ingressantes e concluintes de uma IES de Itararé-SP, visando encontrar as diferenças entre os resultados não satisfatórios de QV. Participaram do estudo acadêmicos de diferentes áreas do conhecimento de uma instituição privada de ensino superior de Itararé - SP. Para a mensuração da qualidade de vida foi utilizado o WHOQOL-Bref, para avaliar o nível de atividade física foi utilizado o Questionário Internacional de Atividade Física (IPAQ) e a satisfação com a imagem corporal foi avaliada por meio de Escala de Silhuetas. Também foram coletadas informações sociodemográficas. A amostra foi composta por 343 acadêmicos, dos quais 175 (51,1\%) eram homens e 168 (48,9\%) eram mulheres, com média de idade de 22,9 \pm 5,6 anos. Foram realizadas análises de diferenças entre 4 grupos comparando cada domínio do WHOQOL-Bref com o sexo, períodos de formação, classes socioeconômicas, níveis de atividade física e satisfação com a imagem corporal. Conclui-se que o sexo, o nível de atividade física e a satisfação com a IC influenciam significativamente na percepção da QV dos estudantes pesquisados. Ao comparar a percepção da QV entre os períodos de formação verificam-se que os estudantes concluintes apresentaram melhores resultados nas facetas relacionadas ao domínio ambiente, quando comparados aos estudantes ingressantes.

PALAVRAS-CHAVE: Qualidade de Vida. Atividade Física. Estudantes.

\section{ABSTRACT}

The objective of the present study was to evaluate the perception of QoL of university students, comparing the information obtained from students and graduates of a HEI of Itararé-SP, in order to find the differences between the unsatisfactory QOL results. The academic study of different areas of knowledge of a private institution of higher education of Itararé-SP - SP participated in the study. To measure the quality of life, the WHOQOL-Bref was used to evaluate the level of physical activity. The International Physical Activity Questionnaire 
(IPAQ) was used and the satisfaction with the body image was assessed by means of a Scale of Silhouettes. Sociodemographic information was also collected. The sample consisted of 343 academicians, of whom 175 (51.1\%) were men and 168 (48.9\%) were women, with a mean age of $22.9 \pm 5.6$ years. Analyzes of differences between groups were performed comparing each WHOQOL-Bref domain with sex, training periods, socioeconomic classes, physical activity levels and body image satisfaction. It was concluded that sex, physical activity level and satisfaction with $\mathrm{BI}$ significantly influence the perception of QoL of the students studied. When comparing the perception of QoL between the training periods, it can be seen that the final students presented better results in the facets related to the environmental domain when compared to the incoming students.

KEYWOORDS: Quality of Life. Physical Activities. Students.

\section{INTRODUÇ̃̃O}

Dados nacionais indicam que o número de Instituições de Ensino Superior (IES) esteve em constante ascensão nos últimos 13 anos, somando 2.391 IES até a conclusão do relatório. Ainda, afirma-se que mais de seis milhões de matrículas foram efetivadas em cursos presenciais, mais de um milhão em cursos de ensino a distância (EAD) e 665 mil em cursos tecnólogos presenciais (SEMESP, 2016). O período de ingresso no ensino superior até a formação é reconhecido como um período de alta tensão, considerando que para os acadêmicos a universidade é sinônimo de acesso ao mercado de trabalho ou manutenção do próprio emprego. Muitas vezes, os acadêmicos mantêm jornada dupla, trabalhando durante um ou dois períodos e estudando em outro período, reduzindo seu tempo livre, o que pode comprometer sua qualidade de vida (QV) (MENDES NETTO, 2013), definida esta pela Organização Mundial da Saúde (OMS) como "a percepção do indivíduo de sua condição na vida, no contexto de sua cultura e dos sistemas de valores em que vive e em relação a suas expectativas, seus padrões e suas preocupações" (FLECK, 2008, p. 25).

Ramis et al. (2012, p. 378) afirmam que o ingresso no meio acadêmico possibilita diversas mudanças na vida do indivíduo, tanto nas relações sociais como na adoção de novos comportamentos, o que normalmente acompanha "situações próprias da adolescência", como alterações físicas e psicológicas, inserindo os universitários em um grupo vulnerável a situações de risco para sua própria saúde.

O estilo de vida, diretamente influenciado pelo comportamento e escolhas feitas em relação aos hábitos do cotidiano, pode ser descrito como a forma de viver de um indivíduo, ou até mesmo de um grupo de pessoas, refletindo diretamente no estado de saúde, o que pode afetar sua percepção da QV (VEIGA; CANTORANI; VARGAS, 2016). Os autores supracitados ainda corroboram com outras pesquisas ao descreverem que a postura adotada e as escolhas feitas diariamente, podem ser positivas ou negativas para o estilo de vida do indivíduo, e que a saúde equilibrada pode influenciar positivamente a QV, que está diretamente relacionada ao cultivo de bons hábitos ao longo da vida.

Neste contexto, avaliar o nível de QV de estudantes universitários torna-se uma abordagem fundamental, visto as constantes mudanças ocorridas no cotidiano que acabam influenciando, tanto na maneira como eles conduzem a sua vida, 
como na valorização de novos comportamentos. Ademais, tais dados podem auxiliar na elaboração de programas da própria instituição, visando a melhoria da QV de seus alunos. Portanto, o objetivo da presente pesquisa foi avaliar a percepção da QV de estudantes universitários, comparando as informações obtidas de ingressantes e concluintes de uma IES de Itararé-SP, visando encontrar as diferenças entre os resultados não satisfatórios de QV.

\section{MATERIAL E MÉTODOS}

A presente pesquisa foi desenvolvida no ano de 2014, nas Faculdades Integradas de Itararé, localizada no município de Itararé-SP. Os acadêmicos investigados pertenciam aos cursos de graduação em Administração, Ciências Contábeis, Direito, Educação Física, História, Pedagogia e Sistemas de Informação. Apenas os alunos ingressantes e concluintes na ocasião da realização da coleta dos dados interessavam à presente pesquisa, visando o objetivo proposto.

Sendo assim, a população-alvo, convidada a participar da pesquisa por conveniência, foi composta por 498 acadêmicos, considerando a população total de alunos ingressantes e concluintes dos cursos pesquisados.

A avaliação da QV foi realizada por meio do WHOQOL-Bref, versão abreviada do questionário elaborado pelo The WHOQOL GROUP da OMS e validado, entre outros públicos, em adultos jovens. Na presente pesquisa, foram analisados os escores obtidos em cada domínio e suas respectivas facetas.

Foram avaliados o nível de atividade física, a satisfação com a imagem corporal (IC) e as informações sociodemográficas, a fim de comparar os dados com os escores obtidos por meio do WHOQOL-Bref.

O nível de atividade física foi avaliado através da versão longa do Questionário Internacional de Atividade Física (IPAQ). Em relação a esta variável, os estudantes foram classificados em ativos fisicamente e insuficientemente ativos. A satisfação com a IC foi avaliada por meio da Escala de Silhuetas de Stunkard, Sorensen e Schulsinger, a qual é composta por nove figuras dispostas em forma crescente, iniciando com a figura de uma silhueta subnutrida até uma silhueta com obesidade grave, e pode ser encontrada na versão masculina e feminina (CAMPANA et al., 2009).

Para o presente estudo, os indivíduos foram orientados a escolher primeiro a figura que representasse o seu corpo atual e posteriormente, deveriam escolher a figura que representasse o corpo que gostariam de ter. O nível de insatisfação dos estudantes foi obtido pela diferença entre as figuras indicas, sendo classificados em satisfeitos e insatisfeitos com a silhueta.

As informações sociodemográficas foram produzidas por meio de um questionário que seguiu os critérios de classificação econômica do Brasil (ABEP, 2010). No caso, foram coletadas informações como: sexo; idade em anos completos; nível econômico (somatório dos pontos por posse de itens, classificando os estudantes em classe baixa ou alta); e período de formação (ingressantes e concluintes).

O tratamento estatístico foi realizado por meio do programa Statistical Package for the Social Sciences (SPSS), versão 20. Na primeira etapa, foi utilizado o teste de normalidade de Kolmogorov-Smirnov para verificar a normalidade dos dados. Em seguida, o teste t para amostras independentes foi utilizado para analisar a diferença entre as médias obtidas de cada grupo nos domínios que compõem a percepção da QV. A variação desses mesmos escores entre os diferentes grupos foi avaliada por meio do teste post hoc de Bonferroni $(\alpha=0,05)$. 
Baseado nas preocupações éticas e metodológicas discutidas nas Diretrizes e Normas Regulamentadoras da pesquisa envolvendo Seres Humanos (Resolução 466/2012), o presente estudo teve o seu projeto de pesquisa encaminhado à Comissão de Ética em Pesquisa em Seres Humanos da Universidade Estadual Ponta Grossa (UEPG), CAAE: 30336514.0.0000.0105, e foi aprovado sob o parecer $n^{\circ} 625.402$ de 30 de abril de 2014.

\section{RESULTADOS}

Da população total foram obtidas 155 perdas, sendo que 143 não concordaram em participar da pesquisa e 12 estudantes foram retirados da pesquisa por terem preenchido os questionários de forma incompleta ou incorretamente em uma ou mais questões. Os 343 acadêmicos participantes da pesquisa, dos quais 175 $(51,1 \%)$ eram homens e $168(48,9 \%)$ eram mulheres, apresentaram média de idade de 22,9 \pm 5,6 anos. A amostra compreendeu 212 estudantes ingressantes e 131 concluintes.

Os dados comparativos entre os sexos e os respectivos resultados em relação aos domínios da QV dos estudantes participantes da presente pesquisa podem ser verificados na Tabela 1:

TABELA 1. Comparação entre sexos e a percepção da QV dos estudantes. Itararé. São Paulo, 2014.

\begin{tabular}{|c|c|c|c|c|c|c|c|}
\hline \multirow{2}{*}{ Dominios } & \multicolumn{3}{|c|}{ Homens $(n=175)$} & \multicolumn{3}{|c|}{ Mulheres $(n=168)$} & \multirow[b]{2}{*}{$\mathrm{p}^{1}$} \\
\hline & $\%$ & Média & DP & $\%$ & Média & $\mathrm{DP}$ & \\
\hline Físico & 73,20 & 15,71 & 2,18 & 70,47 & 15,28 & 2,17 & 0,064 \\
\hline Psicológico & 72,69 & 15,63 & 1,91 & 68,77 & 15,00 & 2,25 & $0,006^{*}$ \\
\hline Relações sociais & 75,76 & 16,12 & 2,73 & 75,20 & 16,03 & 2,71 & 0,759 \\
\hline Ambiente & 62,55 & 14,00 & 1,85 & 59,90 & 13,58 & 2,15 & $0,050^{*}$ \\
\hline $\begin{array}{l}\text { Avaliação global da qualidade de } \\
\text { vida }\end{array}$ & 69,86 & 15,17 & 1,66 & 67,12 & 14,74 & 1,71 & $0,017^{*}$ \\
\hline
\end{tabular}

$\mathrm{Na}$ Tabela 1, ao analisar as facetas que compõem o domínio Psicológico, as facetas em que os homens apresentam melhores resultados são as relacionadas aos sentimentos positivos, IC e sentimentos negativos. Já no domínio Ambiente, as facetas apontadas são as relacionadas aos cuidados de saúde e transporte.

No domínio Físico, as facetas relacionadas à dor e desconforto, energia e fadiga, dependência de medicação ou tratamento, também apresentam uma diferença considerável. No entanto, as médias dos grupos no domínio não foram diferentes de modo significativo. O domínio Relações Sociais não apresentou facetas com diferença significativa entre os sexos.

$\mathrm{Na}$ Tabela 2 podem ser verificados os dados comparativos entre os períodos de formação e os respectivos resultados em relação aos domínios da QV dos estudantes participantes da presente pesquisa: 
TABELA 2. Comparação entre períodos de formação e a percepção da QV dos estudantes. Itararé.

\begin{tabular}{|c|c|c|c|c|c|c|c|}
\hline \multirow{2}{*}{ Domínios } & \multicolumn{3}{|c|}{ Ingressantes $(\mathrm{n}=\mathbf{2 1 2})$} & \multicolumn{3}{|c|}{ Concluintes ( $n=131)$} & \multirow[b]{2}{*}{$\mathrm{p}^{1}$} \\
\hline & $\%$ & Média & DP & $\%$ & Média & $\mathrm{DP}$ & \\
\hline Físico & 71,58 & 15,45 & 2,27 & 72,54 & 15,57 & 2,19 & 0,631 \\
\hline Psicológico & 70,67 & 15,30 & 2,22 & 71,43 & 15,39 & 2,03 & 0,702 \\
\hline Relações sociais & 74,60 & 15,93 & 3,00 & 75,69 & 16,08 & 2,37 & 0,604 \\
\hline Ambiente & 59,81 & 13,56 & 2,04 & 63,00 & 14,03 & 1,94 & $0,039^{*}$ \\
\hline $\begin{array}{l}\text { Avaliação total da qualidade de } \\
\text { vida }\end{array}$ & 67,83 & 14,85 & 1,80 & 69,46 & 15,07 & 1,60 & 0,242 \\
\hline
\end{tabular}

Comparando a percepção da QV entre os períodos de formação verificam-se que os estudantes concluintes apresentaram melhores resultados nas facetas relacionadas aos recursos financeiros, cuidados de saúde, novas informações e habilidades e ambiente físico, quando comparados aos estudantes ingressantes.

Os dados comparativos entre as classes socioeconômicas e os respectivos resultados em relação aos domínios da QV dos estudantes participantes da presente pesquisa podem ser verificados na Tabela 3:

TABELA 3. Comparação entre classes socioeconômicas e a percepção da QV dos estudantes.

\begin{tabular}{|c|c|c|c|c|c|c|c|}
\hline \multirow{2}{*}{ Dominios } & \multicolumn{3}{|c|}{ Baixa $(n=131)$} & \multicolumn{3}{|c|}{ Alta (n=212) } & \multirow[b]{2}{*}{$\mathrm{p}^{1}$} \\
\hline & $\%$ & Média & $\mathrm{DP}$ & $\%$ & Média & $\mathrm{DP}$ & \\
\hline Físico & 72,03 & 15,48 & 2,07 & 71,77 & 15,52 & 2,37 & 0,867 \\
\hline Psicológico & 71,88 & 15,21 & 2,10 & 70,09 & 15,50 & 2,08 & 0,218 \\
\hline Relações sociais & 74,05 & 16,22 & 2,59 & 76,38 & 15,84 & 2,90 & 0,230 \\
\hline Ambiente & 59,49 & 13,97 & 2,08 & 62,34 & 13,51 & 1,86 & $0,037^{*}$ \\
\hline $\begin{array}{l}\text { Avaliação total da qualidade de } \\
\text { vida }\end{array}$ & 68,25 & 14,98 & 1,66 & 68,68 & 14,92 & 1,75 & 0,721 \\
\hline
\end{tabular}

Legenda: ${ }^{1}=$ Valor de $p$ do teste t para amostras independentes; DP = Desvio padrão; ${ }^{*}=$ Diferença significativa ao nível de confiança 0,05 .

Na Tabela 3, estudantes classificados como classe socioeconômica "Alta" apresentam melhores resultados em relação aos estudantes classificados como classe socioeconômica "Baixa", nas facetas relacionadas aos recursos financeiros, recreação e lazer e transporte.

$\mathrm{Na}$ comparação entre a percepção da QV e o nível de atividade física, apresentada na Tabela 4, o grupo fisicamente ativo demonstrou melhores resultados da QV, sobretudo nas facetas relacionadas à energia e fadiga, mobilidade e atividades de vida cotidiana: 
TABELA 4. Comparação entre níveis de atividade física e a percepção da QV dos estudantes. Itararé. São Paulo, 2014

\begin{tabular}{lccccccc}
\hline Domínios & $\begin{array}{c}\text { Ativos fisicamente } \\
(\mathbf{n}=\mathbf{2 1 7})\end{array}$ & \multicolumn{4}{c}{$\begin{array}{c}\text { Insuficientemente } \\
\text { ativos (n=126) }\end{array}$} \\
& $\%$ & Média & DP & $\%$ & Média & DP & P $^{1}$ \\
\hline Físico & 73,03 & 15,68 & 2,22 & 69,87 & 15,17 & 2,08 & 0,036 \\
Psicológico & 71,70 & 15,47 & 2,03 & 69,18 & 15,06 & 2,19 & 0,094 \\
Relações sociais & 75,61 & 16,09 & 2,72 & 75,26 & 16,04 & 2,70 & 0,854 \\
Ambiente & 62,10 & 13,93 & 1,95 & 59,80 & 13,56 & 2,09 & $0,109^{*}$ \\
$\begin{array}{l}\text { Avaliação total da qualidade de } \\
\text { vida }\end{array}$ & 69,52 & 15,12 & 1,70 & 66,79 & 14,68 & 1,66 & 0,021 \\
\hline
\end{tabular}

Legenda: ${ }^{1}=$ Valor de $p$ do teste t para amostras independentes; DP = Desvio padrão; * = Diferença significativa ao nível de confiança 0,05 .

Os dados comparativos entre a satisfação com a IC e os respectivos resultados em relação aos domínios da QV dos estudantes participantes da presente pesquisa podem ser verificados na Tabela 5:

TABELA 5. Comparação entre satisfação com a imagem corporal e a percepção da QV dos estudantes. Itararé. São Paulo, 2014

\begin{tabular}{|c|c|c|c|c|c|c|c|}
\hline \multirow{2}{*}{ Dominios } & \multicolumn{3}{|c|}{$\begin{array}{l}\text { Satisfeitos com } \\
\text { silhueta }(\mathrm{n}=135)\end{array}$} & \multicolumn{3}{|c|}{$\begin{array}{l}\text { Insatisfeitos com a } \\
\text { silhueta }(n=208)\end{array}$} & \multirow[b]{2}{*}{$p^{1}$} \\
\hline & $\%$ & Média & DP & $\%$ & Média & DP & \\
\hline Físico & 72,80 & 15,64 & 2,36 & 70,98 & 15,35 & 2,14 & 0,249 \\
\hline Psicológico & 73,52 & 15,76 & 1,83 & 68,71 & 14,99 & 2,28 & $0,001^{*}$ \\
\hline Relações sociais & 76,79 & 16,28 & 2,57 & 74,48 & 15,91 & 2,78 & 0,217 \\
\hline Ambiente & 61,76 & 13,88 & 2,11 & 60,80 & 13,72 & 1,95 & 0,493 \\
\hline $\begin{array}{l}\text { Avaliação total da qualidade de } \\
\text { vida }\end{array}$ & 70,02 & 15,20 & 1,65 & 67,32 & 14,77 & 1,76 & $0,024^{*}$ \\
\hline
\end{tabular}

$\mathrm{Na}$ Tabela 5 o grupo satisfeito com a silhueta apresenta resultados superiores nas facetas relacionadas a sentimentos positivos, autoestima e IC e aparência.

\section{DISCUSSÃO}

Na comparação da percepção da QV entre sexos, resultados próximos foram encontrados em pesquisa realizada com universitários, na qual se pode constatar que os homens, quando comparados com as mulheres, apresentaram resultados positivos nos domínios Físico, Psicológico e Geral (CIESLAK et al., 2012). Segundo Mendes Netto et al. (2013), há uma tendência para que os homens apresentem melhores resultados quando comparados com as mulheres, haja vista que estas apresentam maior propensão a se preocupar mais com os aspectos relacionados ao desenvolvimento humano, como sentimentos, autoestima, crenças pessoais e a formação da personalidade. Além desses aspectos, as mulheres tendem a praticar menos atividade física que os homens, o que pode ser confirmado pelos resultados do presente estudo. Rechenchosky et al. (2012), ao compararem o estilo de vida adotado pelos universitários, não encontraram associação significativa do estilo de vida geral entre ingressantes e concluintes. Em contrapartida, a pesquisa apresenta resultados sobre relacionamento social e controle do estresse melhor em 
ingressantes e, na dimensão relacionada à nutrição, os resultados foram melhores nos formandos.

Os resultados obtidos na presente pesquisa podem ser justificados ao se comparar as respostas dos estudantes ingressantes e concluintes. Primeiramente nota-se a possibilidade de estágio remunerado que os concluintes possuem, enquanto os ingressantes, via de regra, não. Com relação à faceta "novas informações e habilidades" pode ser fruto tanto dos conhecimentos e experiências vividas na faculdade, como nos campos de estágio e projetos de pesquisa.

Ao ingressar na universidade até o término do curso, novos comportamentos podem ser adquiridos, os quais podem ser positivos ou negativos para a saúde geral, o que pode interferir na percepção da QV do concluinte (RECHENCHOSKY et al., 2012). Na presente pesquisa, provavelmente os comportamentos adquiridos foram positivos, sobretudo quando relacionados ao domínio Ambiente. Nos demais domínios, os concluintes também apresentaram melhores resultados, mas a diferença em relação aos ingressantes não foi significativa.

Ao se comparar a percepção da QV entre as classes socioeconômicas, deve-se considerar que a IES em questão é uma faculdade particular, e que alguns alunos residem em cidades vizinhas. Contudo, alunos enquadrados na classe socioeconômica "Baixa", provavelmente, apresentam menos condições financeiras e tempo - em decorrência do trabalho - para desfrutar de atividades de recreação e lazer. Também é provável que os alunos da classe "Baixa" tentam a utilizar meios de transporte coletivos, o que demanda naturalmente em mais tempo de deslocamento, quando comparados às pessoas que utilizam meios de transporte próprios.

Segundo Nunes et al. (2014), o lazer possui uma definição individual, relacionada com a geração de prazer e satisfação das necessidades pessoais. Em pesquisa realizada com 273 universitários de diversos cursos e Estados brasileiros, os autores avaliaram a satisfação e autonomia nas atividades de lazer entre os sujeitos pesquisados. Na referida pesquisa, $51 \%$ dos estudantes foram classificados como pertencentes às classes econômicas baixa e média, e apresentaram taxas significativamente menores nas atividades de dormir e descansar, e frequentar bares, restaurantes e festas, em comparação aos universitários das classes média alta e alta. Na mesma pesquisa, constatou-se, ainda que, estudantes que não trabalhavam, encontravam-se mais satisfeitos com relação às atividades de lazer.

Ao se analisar o lazer como percepção individual, não se pode afirmar que esta condição seja, necessariamente, proporcional à quantidade de dinheiro que a pessoa possui, visto que existem opções de lazer, como bares, festas e atividades culturais para todas as classes socioeconômicas. Possivelmente, os estudantes enquadrados na classe socioeconômica "Alta", apresentaram melhores resultados em algumas facetas, por receberem ajuda financeira dos pais, ou por receberem maior remuneração no trabalho ou estágio que desenvolvem.

Com relação à atividade física, esta pode se apresentar como uma boa opção para melhorar a QV de estudantes, sendo que, além do gasto energético e sensação de bem-estar, a atividade física pode resultar em um "bom nível de bem-estar biopsicofísico", o que pode contribuir para uma melhor percepção da QV (MENDES NETTO, 2013, p. 52). Na comparação entre a percepção da QV e o nível de atividade física, pesquisadores encontraram uma relação positiva entre atividade física e QV em universitários, ao encontrar um resultado 
satisfatório de QV em mais de $71 \%$ dos homens e mais de $53 \%$ das mulheres classificados como ativos e muito ativos fisicamente (CIESLAK et al., 2012).

Em geral, os homens costumam praticar mais atividade física do que as mulheres, comportamento que pode ser observado desde a infância, quando as meninas preferem brincadeiras com menor gasto energético, como brincar de bonecas, enquanto que os meninos praticam jogos de bola e de aventura (MENDES NETTO, 2013). Na fase jovem e adulta o fato pode ser justificado culturalmente, quando é comum perceber as mulheres gastando muito tempo nos afazeres domésticos, à medida que os homens ocupam-se de atividades físicas mais vigorosas (LANSINI et al., 2017). Esses dados podem ser confirmados na pesquisa de Guedes; Legnani; Legnani (2012), ao avaliar a motivação para a adesão ao exercício físico de 2.380 estudantes da Universidade Estadual de Londrina. A amostra apresentava idade de 18 a 35 anos, e os resultados apontaram que $72 \%$ da amostra feminina não pratica atividade física.

Ao comparar a percepção da QV com a satisfação com a IC, Frank et al. (2016), avaliaram 299 acadêmicos de Educação Física de uma universidade de Santa Catarina, e encontraram uma taxa de $76 \%$ de estudantes insatisfeitos com a IC. A porcentagem entre as mulheres foi mais acentuada, chegando à casa dos $82,5 \%$ de insatisfação. Resultados semelhantes foram encontrados em pesquisa de Rebolho Martins et al. (2012) e Melo et al. (2016), aonde a maioria dos estudantes universitários - de diversas áreas e com média de idade de 20 anos, de universidades de SC e do RS, respectivamente - manifestaram insatisfação com a IC. Supõe-se que o descontentamento das mulheres com relação à IC pode estar relacionado à influência da mídia, que aponta o padrão de beleza feminino, supervalorizando as formas corporais magras. Outro fator que influencia na insatisfação dessa população é o meio sociocultural em que a mulher está inserida (REBOLHO MARTINS et al., 2012).

$\mathrm{Na}$ presente pesquisa, a taxa de insatisfação encontrada foi de $60 \%$. As publicações encontradas acerca do tema relacionam a IC com o sexo, especificamente, o que não foi considerado na presente pesquisa. Porém, as pesquisas disponíveis indicam a associação negativa entre a insatisfação com a IC e a QV, evidenciando a mesma como um problema de saúde pública atual (CLAUMANN et al., 2017).

A presente pesquisa analisou uma amostra de estudantes de uma faculdade particular do interior de São Paulo, com ementas e rotinas específicas dos respectivos cursos e alunos investigados, o que impede, dessa forma, que os resultados aqui apresentados sejam generalizados para todos os estudantes das IES particulares de todo o país. Mais estudos serão necessários para que possam ser aplicadas comparações entre regiões do país e, até mesmo, da macrorregião e microrregião em que se realizou o presente estudo. Entretanto, a pesquisa em pauta possibilita a visualização de uma parcela desse todo. Também serão necessárias novas pesquisas para que se possa inter-relacionar a disponibilidade de tempo livre desses estudantes para fins de uma ampliação da discussão acerca do lazer.

Considerando a escassez de pesquisas científicas envolvendo os instrumentos utilizados neste estudo com a população universitária, sugere-se que novas pesquisas sejam realizadas em universidades próximas e que seja considerada a possibilidade de investigar cada curso de forma específica, para avaliar em que medidas estes precisam de adequações para fins de melhoria da QV de seus respectivos alunos. 


\section{CONSIDERAÇÕES FINAIS}

Os resultados encontrados nesta pesquisa apontam o sexo, o nível de atividade física e a satisfação com a IC, como fatores que influenciam significativamente na percepção da QV dos estudantes ingressantes e concluintes de diferentes cursos de graduação das Faculdades Integradas de Itararé. Observou-se, também, que o domínio que mais apresentou diferença entre cada grupo de comparação, foi o Ambiente, sobretudo nas facetas relacionadas aos cuidados com a saúde, transporte e recursos financeiros.

Os resultados encontrados e as pesquisas citadas corroboram com a necessidade de que os cursos de graduação apresentem em seus programas, conteúdos voltados à adoção de um estilo de vida saudável e valorização de aspectos relacionados à QV do estudante. Investir em programas interdisciplinares de promoção à saúde pode ser um dos caminhos a seguir, somando o conhecimento dos diversos cursos disponibilizados pela instituição na criação de projetos de extensão, para que os alunos possam vir a participar. Outra opção são os programas de assistência ao estudante, os quais são vistos em muitas universidades públicas, com a disponibilização - em baixo custo - de restaurantes universitários, serviços de saúde e moradia para os estudantes da instituição.

AGRADECIMENTOS: Agradecemos à Secretaria de Estado da Saúde de Santa Catarina pela bolsa de estudos para os Residentes e à Fundação de Amparo à Pesquisa e Inovação do Estado de Santa Catarina - FAPESC (Termo de Outorga 2019TR70) pelo apoio. Conceitualmente o termo qualidade de vida, em virtude de sua complexidade

\section{REFERÊNCIAS}

CAMPANA, Angela Nogueira Neves Betanho et al. Avaliação da imagem corporal: instrumentos e diretrizes para a pesquisa. São Paulo: Ed. Phorte; 2009.

CIESLAK, Fabrício et al. Análise da qualidade de vida e do nível de atividade física em universitários. Journal of Physical Education, Maringá, v. 23, n. 2, p. 251-260, 2012.

CLAUMANN, Gaia Salvador et al. Qualidade de vida em acadêmicos ingressantes em cursos de Educação Física. Journal of Physical Education, Maringá, v. 28, n. 1, p. 2824-2834, 2017.

FLECK, Marcelo Pio de Almeida (org). A avaliação de qualidade de vida: guia para profissionais da saúde. Porto Alegre, RS: Ed. Artmed; 2008.

FRANK, Robson et al. Fatores associados à insatisfação com a imagem corporal em acadêmicos de Educação Física. Jornal Brasileiro de Psiquiatria, Rio de Janeiro, v. 65, n. 2, p. 161-7, 2016.

GUEDES, Dartagnan Pinto; LEGNANI, Rosimeide Francisco Santos; LEGNANI, Elto. Motivação para a adesão ao exercício físico: diferenças de acordo com 
experiência de prática. Revista Brasileira de Ciência e Movimento, Distrito Federal, v. 20, n. 3, p. 55-62, 2012.

LANSINI, Luís Carlos et al. Nível de sedentarismo entre estudantes universitários do Rio Grande do Sul e os possíveis fatores associados. Revista O Mundo da Saúde, São Paulo, v. 41, n. 3, p. 267 - 274, 2017.

MELO, Priscila Evangelista et al. Percepção da autoimagem corporal de universitários. Cinergis, Santa Cruz do Sul, v. 17, n. 3, p. 208-213, 2016.

MENDES-NETTO, Raquel Simões et al. Nível de atividade física e qualidade de vida de estudantes universitários da área de saúde. Revista de Atenção à Saúde, São Caetano do Sul, v. 10, n. 34, p. 47-55, 2013.

NUNES, Maiana Farias Oliveira et al. Satisfação e autonomia nas atividades de lazer entre universitários. Psicologia: teoria e prática, São Paulo, v. 16, n. 1, p. 91-103, 2014

RAMIS, Thiago Rozales et al. Tabagismo e consumo de álcool em estudantes universitários: prevalência e fatores associados. Revista Brasileira de Epidemiologia, São Paulo, v. 15, n. 2, p. 376-385, 2012.

REBOLHO MARTINS, Cilene et al. Insatisfação com a imagem corporal e fatores associados em universitários. Estudos de Psicologia, Online, v. 17, n. 2, p. 241-246, 2012.

RECHENCHOSKY, Leandro et al. Estilo de vida de universitários calouros e formandos de educação física de uma universidade pública do centro-oeste brasileiro. Coleção Pesquisa em Educação Física, São Paulo, v. 11, n. 5, p. 47-54, 2012.

SEMESP. Mapa do Ensino Superior no Brasil. 2015. Disponível em <http://convergenciacom.net/pdf/mapa-ensino-superior-brasil-2015.pdf>. Acesso em: 20 setembro 2016.

VEIGA, Carolina; CANTORANI, José Roberto Herrera; VARGAS, Leandro Martinez. Qualidade de vida e alcoolismo: um estudo em acadêmicos de licenciatura em educação física. Conexões, Campinas, v. 14, n. 1, p. 20-34, 2016. 